\title{
Comparison of treatment strategies for patients with limited-stage small cell lung cancer who received chemotherapy
}

\author{
Shenhai Wei ${ }^{1 \#}$, Bo Wei ${ }^{2 \#}$, Jintao Tian $^{1}$, Xiaoping Song ${ }^{1}$, Bingqun $\mathrm{Wu}^{1}$, Pengcheng $\mathrm{Hu}^{1}$ \\ ${ }^{1}$ Department of Thoracic Surgery, The First Hospital of Tsinghua University, Beijing 100016, China; ${ }^{2}$ Department of Thoracic Surgery, Beijing \\ Tiantan Hospital, Capital Medical University, Beijing 100050, China \\ Contributions: (I) Conception and design: S Wei, B Wei; (II) Administrative support: J Tian, S Wei; (III) Provision of study materials or patients: \\ S Wei; (IV) Collection and assembly of data: X Song, B Wu, P Hu; (V) Data analysis and interpretation: S Wei, B Wei; (VI) Manuscript writing: All \\ authors; (VII) Final approval of manuscript: All authors. \\ "These authors contributed equally to this work. \\ Correspondence to: Dr. Shenhai Wei. Department of Thoracic Surgery, The First Hospital of Tsinghua University, No. 6, the First Road, Jiuxiaoqiao, \\ Chaoyang District, Beijing 100016, China. Email: weishenh@qq.com.
}

Background: The standard care for limited-stage small cell lung cancer (SCLC) is unclear. The purpose of this study is to compare the outcome for patients receiving chemotherapy alone, chemotherapy plus surgery (CS), chemotherapy plus radiation (CR), or chemotherapy plus surgery and radiation (CSR) for limited-stage SCLC.

Methods: Patients with T1-4N0-2M0 SCLC who received chemotherapy from 2004 to 2014 were retrieved from the Surveillance, Epidemiology, and End Results (SEER) database. The overall survival (OS) of these patients, stratified by different stage, was compared in accordance to the method of receiving different treatments using Kaplan-Meier method and Cox regression analysis.

Results: A total of 7,204 patients were included, where 1,347 (18.7\%) patients received chemotherapy alone, 296 (4.1\%) undergone CS, 5,296 (73.5\%) patients were subjected to CR and 267 (3.7\%) patients were managed by the three combination of CSR. Chemotherapy alone was associated with the worst survival in comparison to the other two method of combination i.e., chemotherapy with radiation or surgery. When compared with CR, CS had no survival benefit in patients with stage in excess of T1-2N0 disease, but was associated with improved 5-year OS in patients with T1-2N0 disease, which ranged from $29.1 \%$ to $54.3 \%$ $(\mathrm{P}<0.001)$. For patients with T1-2N2 disease who received CSR demonstrated superior OS over those who received $C R(P=0.004)$ or $C S(P=0.036)$. Cox regression analysis showed $C S$ was associated with improved OS when compared with CR in patients with N0 disease (HR, 0.54; 95\% CI, 0.43-0.68; P=0.000) and CSR was associated with better OS in comparison with CR in patients with N2 disease (HR, 0.71; 95\% CI, 0.55$0.93 ; \mathrm{P}=0.013)$.

Conclusions: Patients with limited-stage SCLC can benefit from local treatment such as surgery, radiation, and surgery plus radiation. For patients with N0 disease, CS was associated with improved survival in comparison to CR. If N2 was identified after surgery, radiation may be added to improve OS.

Keywords: Small cell lung cancer (SCLC); surgical resection; Surveillance, Epidemiology, and End Results (SEER); overall survival (OS)

Submitted Aug 18, 2019. Accepted for publication Nov 12, 2019.

doi: $10.21037 /$ tcr.2019.12.13

View this article at: http://dx.doi.org/10.21037/tcr.2019.12.13 


\section{Introduction}

Lung cancer is the most common global cancer among all cancers, and small cell lung cancer (SCLC) comprises approximately $14 \%$ of all lung cancers $(1,2)$. SCLC behaves differently from non-small cell lung cancer (NSCLC) and is noteworthy for its early development, rapid doubling time, high growth fraction, with and its widespread metastases. Most patients with SCLC present with hematogenous metastasis, and only one-third of patients present with limited disease at diagnosis (3).

The standard treatment for patients with limited-stage SCLC is concurrent administration of chemoradiotherapy, which was illustrated from historical researches where there was survival improvement with radiation in comparison to surgery $(4,5)$. However, positive experiences with surgery in patients with early stage SCLC continue to be reported, including some population-based studies (6-14). In the latest National Comprehensive Cancer Network (NCCN) guidelines, surgery combined with chemotherapy has been recommended for patients with stage T1-2N0 SCLC (3). However, up to date, few studies have compared different treatments such as: as a single treatment, or in combination for example, chemotherapy plus radiation (CR), chemotherapy plus surgery (CS), and chemotherapy plus surgery and radiation (CSR) for limited-stage SCLC. In this present study, we aimed to make such a comparison for limited-stage SCLC using data from the Surveillance, Epidemiology, and End Results (SEER) Program of the National Cancer Institute (NCI).

\section{Methods}

The SEER Program collects information that includes stages of cancer at the time of diagnosis and patients' survival data, which was extracted from 18 populationbased registered cancer institutes that cover approximately $28 \%$ of the US population (1). We used SEER*Stat version 8.3.4 software to extract data in the client-server mode. This study was approved by the review board of the First Hospital of Tsinghua University and the number of the approval was 2019-06.

\section{Patient selection}

The selection criteria included patients' that are pathologically confirmed with SCLC as their first or only primary malignancy, from 2004 to 2013. Only patients that were included in this study were coded as stage N0-2 [according to the 6th edition of the American Joint Committee on Cancer (AJCC)] and M0 (according to the AJCC 8th edition (15) criteria: no distant metastasis, no pleural or pericardial nodules and malignant effusion) and where chemotherapy had been administered. Patients excluded from this study were those who had received other surgical treatment except wedge resection, segmentectomy, lobectomy, bilobectomy or pneumonectomy. All patients without full information regarding tumor size, tumor extension, or radiation were excluded. Figure 1 shows the detailed description of patients' selection process. Ultimately, our study comprised of 7,204 patients.

\section{Data preparation}

The data that was extracted for this study included age, sex, race, year of diagnosis, tumor size, tumor extension, separate nodules of the ipsilateral lung, $\mathrm{N}$ stage (based on the AJCC 6th edition), surgical procedure, and radiation. The T stage, based on the AJCC 8th edition, was derived according to tumor size, tumor extension, and separate nodules in the ipsilateral lung (15). Surgical types were categorized as sublobectomy (including wedge resection and segmentectomy), lobectomy (including bilobectomy), or pneumonectomy. Radiation referred to thoracic beam radiation, and in CSR group, only postoperative radiation was included. We categorized patients' ages into four groups as follows: $<60,60-69,70-79$, and $\geq 80$ years. Patients' races were combined into a dichotomized variable in the analysis. The year of diagnosis was grouped as follows: 2004-2006, 2007-2010, and 2011-2014. The endpoints of this study were the overall survival (OS). OS was defined as the time period from diagnosis to death from any cause.

\section{Statistics analysis}

Survival curves were generated using the Kaplan-Meier method, and for comparative differences we used the log-rank test. For Multivariable analyses we used a Cox regression model. The hazard ratio (HR) with $95 \%$ confidence interval (CI) was estimated. All reported P values were two-sided and a value $<0.05$ was considered significant. All analyses were conducted using Stata/MP 14.0 for Windows (StataCorp LP, College Station, TX, USA). 


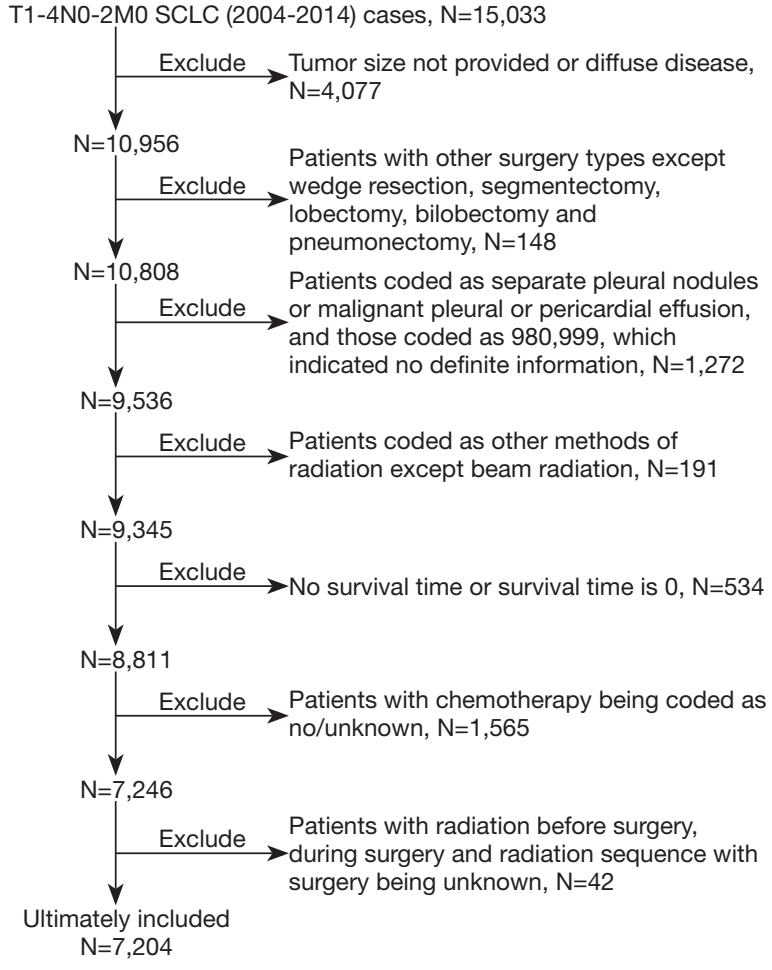

Figure 1 Patient selection in the study.

\section{Results}

\section{Patients' characteristics}

The patient cohort $(\mathrm{n}=7,204)$ in this study consisted of 3,315 men $(46.0 \%)$ and 3,889 women $(54.0 \%)$ with a mean age of $65.4 \pm 9.7$ years (range, 29-93 years). The last followup occurred in December 2014, and the median follow-up duration was 15 months (range, 1-131 months). A total of 5,244 patients $(72.8 \%)$ died during the follow-up period, and the median OS was 18 months.

Patients and tumor characteristics with different treatment combinations are shown in Table 1. There were $294(4.1 \%)$ patients that received CS, a total of 5,296 (73.5\%) patients had CR and 267 (3.7\%) patients undergone CSR, respectively. A higher proportion of CR was noted in each subgroup of patients compared to other treatment combinations. Surgery was offered only for a small number of patients $(\mathrm{n}=561,7.8 \%)$ and most of the procedure that was implemented is lobectomy $(\mathrm{n}=381$, $67.9 \%$ ) (Table 1).

Analysis for all patients' survival showed that those who received chemotherapy alone had the poorest survival (median OS of 10 months and 5-year OS rate of $6.1 \%$ ).
Treatment combination of CS offered a significant and better survival compared with CR (5-year OS, 42.6\% for $\mathrm{CS}$ versus $20.1 \%$ for $\mathrm{CR}, \mathrm{P}<0.001)$ and have similar OS with CSR (Figure 2). After stratified by stage, CS was associated with better survival only in patients with T1-2N0 disease when compared with CR (Figure $3 A$ ). There was no survival benefit for CSR compared to CS except in patients with T1-4N2 disease (Figure 3). Patients undergone CSR were also associated with improved survival compared with those who underwent CR in patients with T1-2N2 disease (Figure 3E).

Lobectomy was associated with better survival when compared with sublobectomy (5-year OS, $46.9 \%$ for lobectomy versus $28.0 \%$ for sublobectomy, $\mathrm{P}<0.001$ ). Survival of patients who underwent pneumonectomy did not differ from those who underwent sublobectomy and lobectomy (Figure 4).

On Cox regression analysis, CS was associated with improved survival in N0 disease as compared to CR, and CSR was associated with improved survival in N2 disease. Chemotherapy alone was associated with increased risk of death across the $\mathrm{N} 0$ to $\mathrm{N} 2$ stage $(\mathrm{P}=0.000$, Table 2).

\section{Discussion}

In this study, chemotherapy alone was associated with the worst survival when compared with the addition of local treatment such as radiation, surgery or surgery plus postoperative radiation except in patients with T3-4N2 disease. In patients with T3-4N2 disease, adding radiation instead of surgery to chemotherapy could improve survival. Our findings provided further evidence to support the use of local treatment for limited stage SCLC as recommended by NCCN guidelines (3).

During univariate analysis for all patients included in this study, CS was associated with improved OS when compared with CR. However, the survival benefit of CS was obviously only in patients with T1-2N0 disease. For patients with T34N0 disease, the survival benefit of CS over CR was not significant. This result was consistent with previous studies and recommendations by the NCCN guidelines $(3,11,13)$.

For patients with SCLC T1-2N1 disease, there was no significant difference in the OS for patients who received CS when compared with those who received CR. For those who with T3-4N1 disease, the survival benefit of CS over CR seemed to be significant (Figure 3D); however, the number of patients who received CS was small $(n=7)$. The result should be interpreted cautiously. The 
Table 1 Baseline characteristic of patients

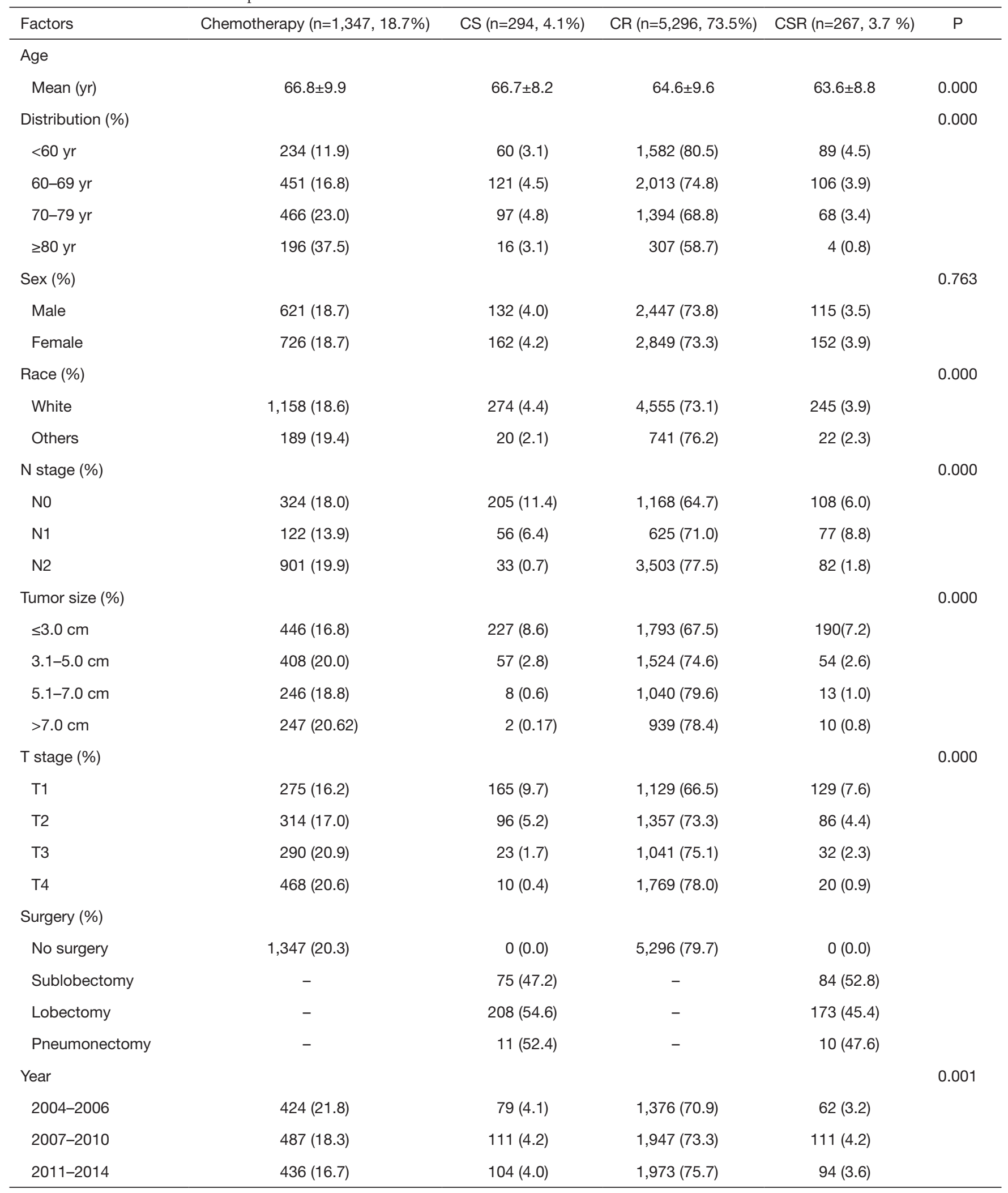

$\mathrm{CR}$, chemotherapy plus radiation; CS, chemotherapy plus surgery; CSR, chemotherapy plus surgery and radiation. 


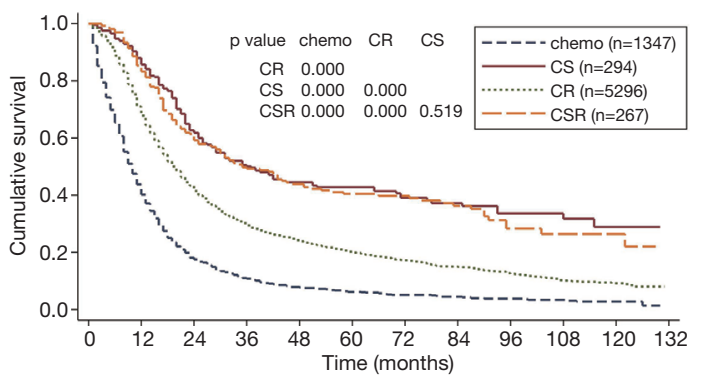

Figure 2 Survival curves according to various treatment combinations in the cohort study. The median OS was 10 months for chemotherapy alone, 20 months for CR, 37 months for CS, and 35 months for CSR $(\mathrm{P}<0.001)$. chemo, chemotherapy alone; $\mathrm{CS}$, chemotherapy plus surgery; $\mathrm{CR}$, chemotherapy plus radiation; CSR, chemotherapy plus surgery and radiation.

results were different from the previous study that was performed by Yang et al., which was a population-based study and used National Cancer Database (NCBD) (16). In that study, surgery plus adjuvant chemotherapy with or without radiation was associated with improved 5 -year survival from $26.3 \%$ to $31.4 \%$ compared with the concurrent chemoradiation for T1-3N1M0 SCLC. The reason for the difference is uncertain. However, in this study maybe the small number of patients with $\mathrm{N} 1$ disease and the different database being used could be the underlying reasons. Further researches with prospective nature are needed on this issue.

In our study results showed no significant difference in the OS between groups of CSR and CS in patients with N0 and N1 disease. However, for patients with T14N2 disease, CSR was associated with improved survival compared with CS. The findings were interestingly parallel to the retrospective studies performed for postoperative radiation in NSCLC settings (17-19). Results indicated that it is reasonable to administer postoperative radiation to patients who are identified as N2 disease after surgery. This result was similar to the study that was performed by Wong et al., where they used the NCDB, and the study that was performed by Schreiber et al., and also they used SEER database $(8,20)$. In the study by Wong et al., the postoperative radiation was associated with improved survival in patients with $\mathrm{N} 2$ disease, but detrimental to patients with $\mathrm{N} 0$ disease and this is most probably because of the early stage of the disease where less invasiveness of the tumor, which might have affected the normal cells more than the tumor cells. In our study, there was no survival deterioration observed in patients with $\mathrm{N} 0$ disease during the postoperative radiation, as well for N1 disease, which was consistent with the results of another population-based study by Schreiber $e t$ al. that used SEER database (8). The reason for the difference between our study and the study by Wong et al., is unclear. Different database with patients' selection bias may be the underlying reason.

It was noted in this study that patients with T1-2N2 disease who received CSR was associated with improved survival when compared with those who received CR. This result must be interpreted cautiously. Stages showed more clinical indications or features for patients who received CR and more pathological for those who received CSR. Clinical T1-2N2 disease may be more advanced than pathological T1-2N2 disease and thus patients with pT1-2N2 disease who received CSR was associated with improved survival rate. Further prospective studies are needed to elucidate this issue.

In this study, surgery did not demonstrate survival benefit when compared with radiation for patients with T3-4N0 disease. For T3-4N1 and T3-4N2 disease, the difference of survival for CS groups and CR groups seemed to be significant; however, the number of patients in CS groups were both small ( $\mathrm{n}=7$ and 5, respectively). The results should be interpreted cautiously. Furthermore, in patients with T3-4N2 disease, patients who received CS were seemed to be associated with worst OS when compared with CR. These findings meant that surgery should not be recommended for patients with advanced $\mathrm{T}$ stage. Future prospective studies are needed to elucidate this issue.

Multivariate analysis with Cox regression model demonstrated improved survival for CS and CSR when compared with CR in patients with N0 disease. Patients with N2 disease who received CSR was associated with improved survival. In patients with $\mathrm{N} 1$ disease, there was no significant difference for the effect of CS, CR or CSR on survival. For all patients, chemotherapy alone offered worst survival when compared with CR.

Chemotherapy is recommended by the NCCN guidelines for all SCLC patients including the resected early stage as long as they are fit enough due to the higher sensitivity of SCLC to chemotherapy $(3,6)$. We presumed that most patients who did not undergo chemotherapy in SEER database may have been too sick to receive chemotherapy. So, we set chemotherapy as one of the inclusion criterions for this study to reduce the selection bias. To our knowledge, this study was the first one to explore the effect of different treatment combination 

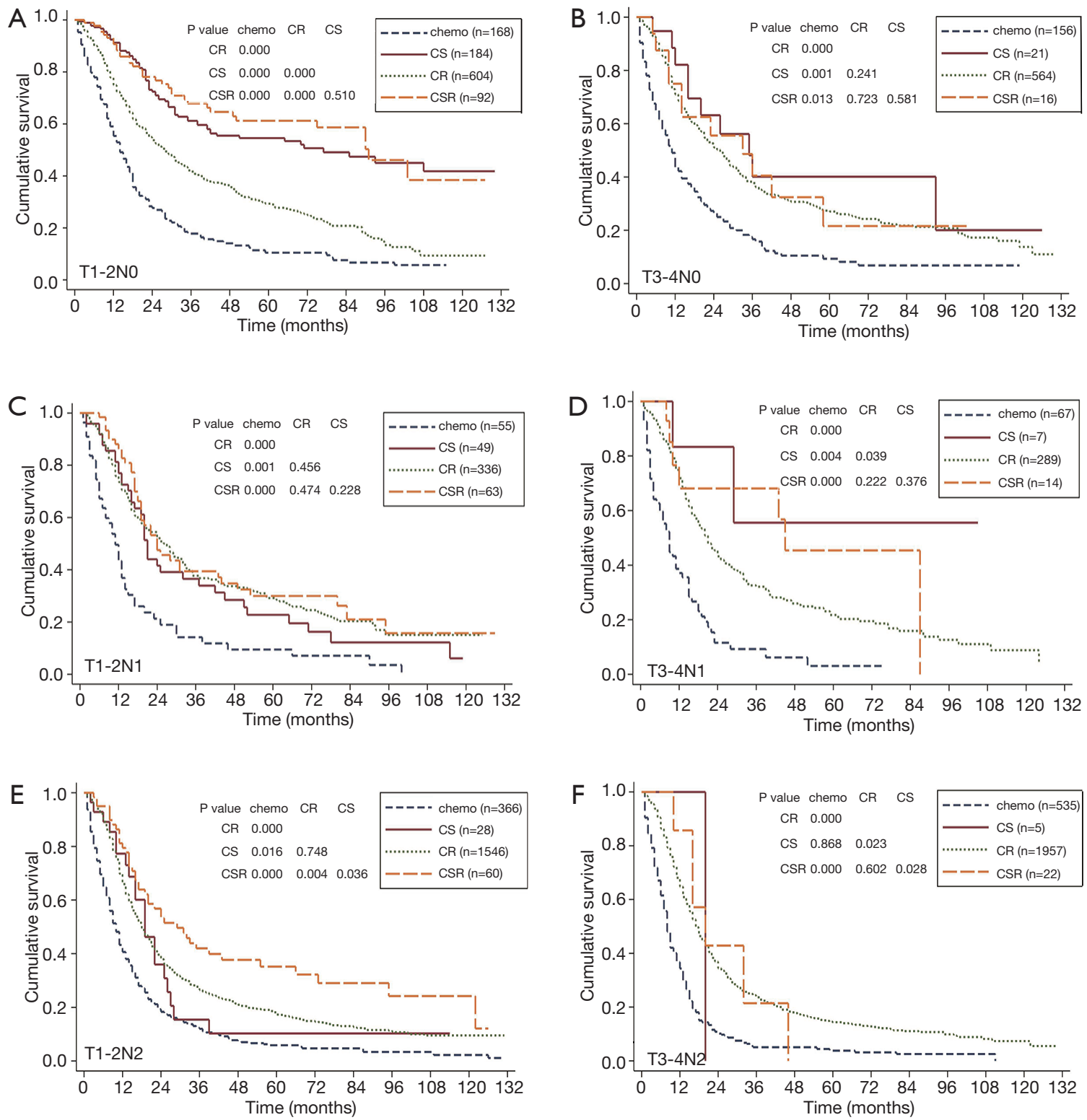

Figure 3 Survival curves according to various treatment combinations in different stage groups. (A) Survival curves according to various treatment combinations in T1-2N0 patients. The median OS was 14 months for chemotherapy alone, 28 months for CR, 77 months for CS, and 91 months for CSR $(\mathrm{P}<0.001)$. (B) Survival curves according to various treatment combinations in T3-4N0 patients. The median OS was 11 months for chemotherapy alone, 26 months for CR, 35 months for CS, and 33 months for CSR (P<0.001). (C) Survival curves according to various treatment combinations in T1-2N1 patients. The median OS was 11 months for chemotherapy alone, 27 months for CR, 21 months for CS, and 24 months for CSR $(\mathrm{P}<0.001)$. (D) Survival curves according to various treatment combinations in T3-4N1 patients. The median OS was 9 months for chemotherapy alone, 21 months for CR, and 45 months for CSR. Median survival for CS did not reach $(\mathrm{P}<0.001)$. (E) Survival curves according to various treatment combinations in $\mathrm{T} 1-2 \mathrm{~N} 2$ patients. The median OS was 10 months for chemotherapy alone, 18 months for CR, 19 months for CS, and 29 months for CSR $(\mathrm{P}<0.001)$. (F) Survival curves according to various treatment combinations in T3-4N2 patients. The median OS was 8 months for chemotherapy alone, 17 months for CR, 12 months for CS, and 16 months for CSR $(\mathrm{P}<0.001)$. chemo, chemotherapy alone; CS, chemotherapy plus surgery; CR, chemotherapy plus radiation; CSR, chemotherapy plus surgery and radiation. 


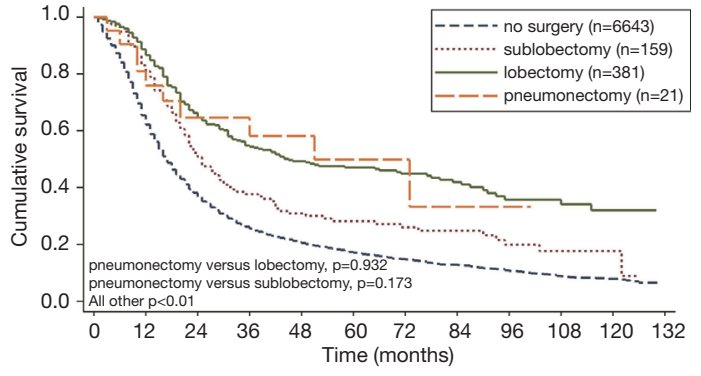

Figure 4 Survival curves according to surgical types. The median OS was 17 months for no surgery, 25 months for sublobectomy, 45 months for lobectomy, and 51 months for pneumonectomy $(\mathrm{P}<0.001)$. including chemotherapy using SEER database for limitedstage SCLC. Furthermore, the stages in this study were based on eighth edition of AJCC staging system. With the inclusion of the details regarding the newest staging system and chemotherapy, this study can reflect a situation much closer to the current clinical practice than the previous SEER-based studies.

However, there are some limitations for this populationbased study. The retrospective reported data were subject to a selection bias and potential errors in data entry. The nodal staging for patients who did not receive surgery may be more advanced than those who received surgery

Table 2 Cox regression analysis of OS including treatment combination for different $\mathrm{N}$ stage

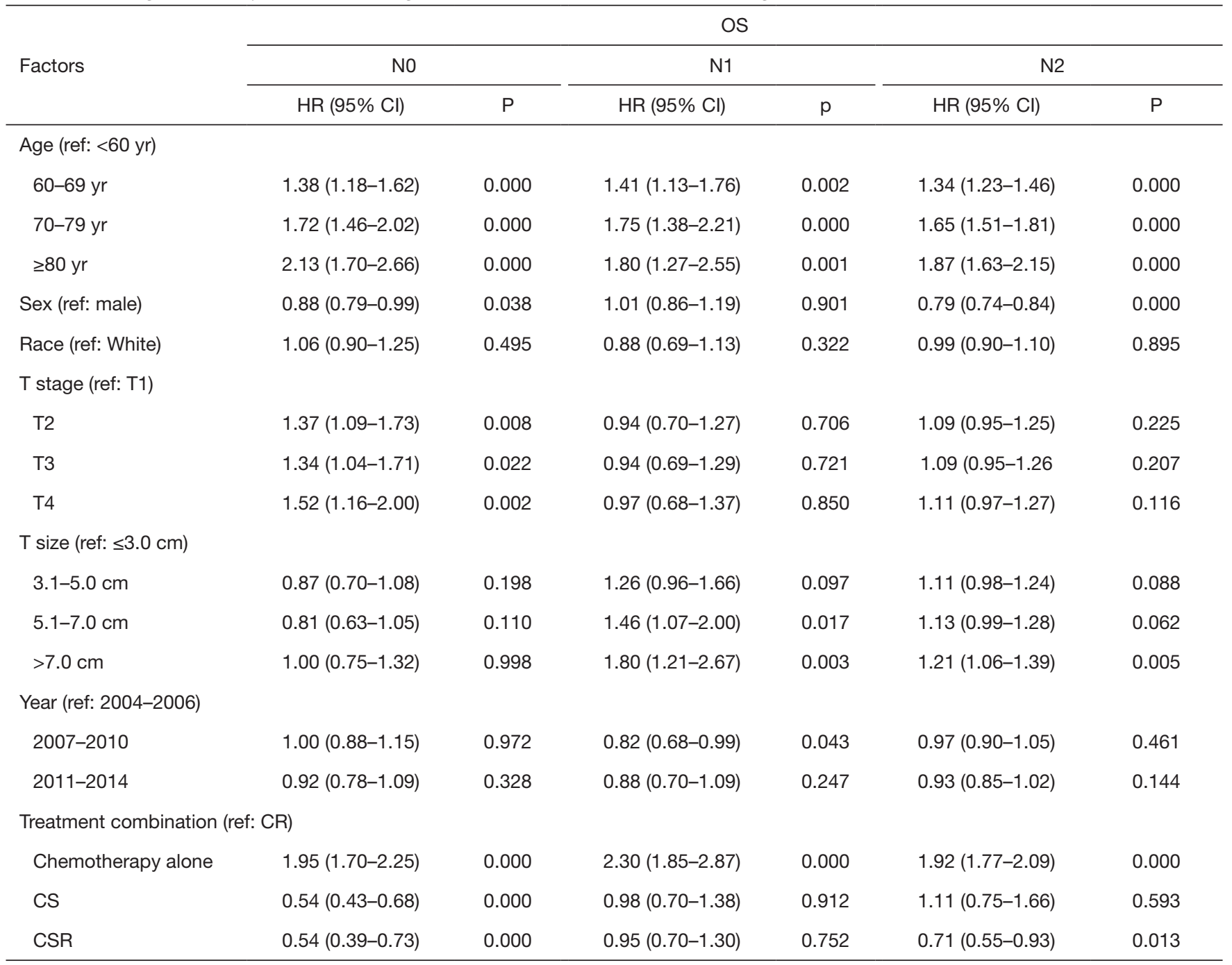

OS, overall survival; HR, hazard ratio; $\mathrm{Cl}$, confidence interval; CR, chemotherapy plus radiation; CS, chemotherapy plus surgery; CSR, chemotherapy plus surgery and radiation. 
as aforementioned. That would have biased the results in favor for surgery. Other limitations in this study include the lack of information regarding the performance status and comorbidities. Poor performance status or severe comorbidities led to nonsurgical treatment and, therefore, most likely had biased the results. In addition, there was no information about the surgical margin status and the postoperative complications. These two confounders would have biased the results towards the null hypothesis. Lastly, there was no information about the adjuvant brain radiation in SEER database. Previous researches demonstrated that patients with limited-stage SCLC who had good response to initial therapy, prophylactic cranial irradiation (PCI) decreased brain metastasis and increased OS (6,21-23). Absent of information for PCI may have led to skewed results of this study.

In conclusion, adding a combination of local treatment for limited stage SCLC can provide survival benefit when compared with chemotherapy alone. In patients with T1$2 \mathrm{~N} 0$ disease, CS was associated with better OS when compared with $\mathrm{CR}$, and postoperative radiation that was added showed no survival improvement. If $\mathrm{N} 2$ disease was identified after surgery, postoperative thoracic radiation may be used to improve OS. For N1 disease, surgery was not recommended when compared to radiation. Owing to the retrospective nature of this study, evidence on a prospective basis will be required to further clarify the optimal treatment combination for limited stage SCLC.

\section{Acknowledgments}

We would like to thank Dr. Hassan Dib for his contribution in proofreading this manuscript.

Funding: None.

\section{Footnote}

Conflicts of Interest: The authors have completed the ICMJE uniform disclosure form (available at http://dx.doi. org/10.21037/tcr.2019.12.13). The authors have no conflicts of interest to declare.

Ethical Statement: The authors are accountable for all aspects of the work in ensuring that questions related to the accuracy or integrity of any part of the work are appropriately investigated and resolved. This study was approved by the review board of the First Hospital of
Tsinghua University and the number of the approval was 2019-06.

Open Access Statement: This is an Open Access article distributed in accordance with the Creative Commons Attribution-NonCommercial-NoDerivs 4.0 International License (CC BY-NC-ND 4.0), which permits the noncommercial replication and distribution of the article with the strict proviso that no changes or edits are made and the original work is properly cited (including links to both the formal publication through the relevant DOI and the license). See: https://creativecommons.org/licenses/by-nc$\mathrm{nd} / 4.0 /$.

\section{References}

1. Howlader N NA, Krapcho M, Miller D, Bishop K, Kosary CL, Yu M, Ruhl J, Tatalovich Z, Mariotto A, Lewis DR, Chen HS, Feuer EJ, Cronin KA (eds) . SEER Cancer Statistics Review, 1975-2014, National Cancer Institute. Bethesda, MD, Available online: https://seer.cancer.gov/ csr/1975_2014/, based on November 2016 SEER data submission, posted to the SEER web site, April 2017. 2017.

2. Siegel RL, Miller KD, Jemal A. Cancer Statistics, 2017. CA Cancer J Clin 2017;67:7-30.

3. National Comprehensive Cancer Network. National Comprehensive Cancer Network. NCCN Clinical Practice Guidelines in Oncology---Small Cell Lung Cancer Version 1. 2019. Available online : http://www. nccn.org/professionals/physician_gls/PDF/sclc.pdf. 2019.

4. Fox W, Scadding JG. Medical Research Council comparative trial of surgery and radiotherapy for primary treatment of small-celled or oat-celled carcinoma of bronchus. Ten-year follow-up. Lancet 1973;2:63-5.

5. Lad T, Piantadosi S, Thomas P, et al. A prospective randomized trial to determine the benefit of surgical resection of residual disease following response of small cell lung cancer to combination chemotherapy. Chest 1994;106:320S-3S.

6. Yang CF, Chan DY, Speicher PJ, et al. Role of Adjuvant Therapy in a Population-Based Cohort of Patients With Early-Stage Small-Cell Lung Cancer. J Clin Oncol 2016;34:1057-64.

7. Yu JB, Decker RH, Detterbeck FC, et al. Surveillance epidemiology and end results evaluation of the role of surgery for stage I small cell lung cancer. J Thorac Oncol 2010;5:215-9. 
8. Schreiber D, Rineer J, Weedon J, et al. Survival outcomes with the use of surgery in limited-stage small cell lung cancer: should its role be re-evaluated? Cancer 2010;116:1350-7.

9. Wakeam E. Surgery for small cell lung cancer in the modern era: opportunities and challenges. J Thorac Dis 2017;9:E1145-7.

10. Zhang J, Li S, Chen X, et al. Retrospective study of surgery versus non-surgical management in limited-disease small cell lung cancer. Thorac Cancer 2014;5:405-10.

11. Combs SE, Hancock JG, Boffa DJ, et al. Bolstering the case for lobectomy in stages I, II, and IIIA small-cell lung cancer using the National Cancer Data Base. J Thorac Oncol 2015;10:316-23.

12. Grossman RA, Pedroso FE, Byrne MM, et al. Does surgery or radiation therapy impact survival for patients with extrapulmonary small cell cancers? J Surg Oncol 2011;104:604-12.

13. Yang CJ, Chan DY, Shah SA, et al. Long-term Survival After Surgery Compared With Concurrent Chemoradiation for Node-negative Small Cell Lung Cancer. Ann Surg 2018;268:1105-12.

14. Weksler B, Nason KS, Shende M, et al. Surgical resection should be considered for stage I and II small cell carcinoma of the lung. Ann Thorac Surg 2012;94:889-93.

15. Detterbeck FC, Boffa DJ, Kim AW, et al. The Eighth Edition Lung Cancer Stage Classification. Chest 2017;151:193-203

16. Yang CJ, Chan DY, Speicher PJ, et al. Surgery Versus Optimal Medical Management for N1 Small Cell Lung Cancer. Ann Thorac Surg 2017;103:1767-72.

Cite this article as: Wei S, Wei B, Tian J, Song X, Wu B, Hu P. Comparison of treatment strategies for patients with limitedstage small cell lung cancer who received chemotherapy. Transl Cancer Res 2020;9(2):818-826. doi: 10.21037/tcr.2019.12.13
17. Wei S, Xie M, Tian J, et al. Propensity score-matching analysis of postoperative radiotherapy for stage IIIA-N2 non-small cell lung cancer using the Surveillance, Epidemiology, and End Results database. Radiat Oncol 2017;12:96.

18. Robinson CG, Patel AP, Bradley JD, et al. Postoperative radiotherapy for pathologic N2 non-small-cell lung cancer treated with adjuvant chemotherapy: a review of the National Cancer Data Base. J Clin Oncol 2015;33:870-6.

19. Lee HW, Noh OK, Oh YT, et al. Radiation TherapyFirst Strategy After Surgery With or Without Adjuvant Chemotherapy in Stage IIIA-N2 Non-Small Cell Lung Cancer. Int J Radiat Oncol Biol Phys 2016;94:621-7.

20. Wong AT, Rineer J, Schwartz D, et al. Assessing the Impact of Postoperative Radiation Therapy for Completely Resected Limited-Stage Small Cell Lung Cancer Using the National Cancer Database. J Thorac Oncol 2016;11:242-8.

21. Yang Y, Zhang D, Zhou X, et al. Prophylactic cranial irradiation in resected small cell lung cancer: A systematic review with meta-analysis. J Cancer 2018;9:433-9.

22. Farris MK, Wheless WH, Hughes RT, et al. LimitedStage Small Cell Lung Cancer: Is Prophylactic Cranial Irradiation Necessary? Pract Radiat Oncol 2019;9:e599-607.

23. Kim TG, Pyo H, Ahn YC, et al. Role of prophylactic cranial irradiation for elderly patients with limiteddisease small-cell lung cancer: inverse probability of treatment weighting using propensity score. J Radiat Res 2019;60:630-8. 